\title{
Video Annotation for Immersive Journalism using Masking Techniques
}

\author{
João Meira*, João Marques ${ }^{\dagger}$, João Jacob, Rui Nóbrega, Rui Rodrigues, António Coelho, and A. Augusto de Sousa \\ FEUP - Faculdade de Engenharia da Universidade do Porto \\ INESC TEC - Instituto de Engenharia de Sistemas e Computadores, Tecnologia e Ciência \\ Email: $\left\{{ }^{*}\right.$ ee11081, ${ }^{\dagger}$ up201106011, joao.jacob, ruinobrega, rui.rodrigues, acoelho, augusto.sousa $\} @$ fe.up.pt
}

\begin{abstract}
This paper proposes an interactive annotation technique for $360^{\circ}$ videos that allows the use of traditional video editing techniques to add content to immersive videos. Using the case study of immersive journalism the main objective is to diminish the entry barrier for annotating $360^{\circ}$ video pieces, by providing a different annotation paradigm and a set of tools for annotation. The spread of virtual reality systems and immersive content has been growing substantially due to technological progress and cost reductions in equipment and software. From all the technologies employed in virtual reality systems, $360^{\circ}$ video is one that currently presents unique conditions to be widely used by various industries - especially for communication purposes. From the various areas that can benefit from the usage of virtual reality systems, the communication field is one that requires innovation in the way that narratives are built, especially in virtual reality systems. In the case of immersive journalism, $360^{\circ}$ video technology is currently one of the most used mediums by several media outlets. This kind of news content, whose innovative role should be highlighted, is still being studied in the field of journalism, needing a clearly defined set of rules and good practises. In order to improve the introduction of virtual elements in the $360^{\circ}$ videos this paper proposes a set of annotation paradigms for 1) Media information display and 2) Narrative and attention focusing. In this paper we present a list of possible techniques that solve the problem of immersive annotation, as well as a description of a prototype that was developed to test these concepts. The prototype implements an annotation technique based on masked videos and the extension of standard subtitle file formats. Finally a fast-track user study was developed to evaluate the acceptance of the visualisation techniques and to refine the set of tools.

Index Terms- $360^{\circ}$ Video, Virtual Reality, Immersive Journalism, Visualization, Annotation, Interaction, Computer Graphics.
\end{abstract}

\section{INTRODUCTION}

Currently, $360^{\circ}$ journalist pieces follow a traditional single camera approach where the camera is located in the centre of the scenario and the journalist is the front part of the scene. This traditional narrative fails to grasp the full capabilities of $360^{\circ}$ videos where the user can interactively look in any direction and ignore the journalist or the topic that $\mathrm{s} / \mathrm{he}$ wants to show or focus.

This apparent additional freedom for the viewer reduces the storytelling narrative ability of the producer. In the long term the value of the journalistic piece may diminish if $s / h e$ fails

978-1-5090-5387-2/16/\$31.00 2016 IEEE to create a compelling story that guides the viewer through the $360^{\circ}+$ time scenario. It is then required to create new paradigms of narrative storytelling that take into account the affordances of the new virtual reality headsets and $360^{\circ}$ videos. These narrative paradigms should take into consideration the user's attention focus, his field of view (FOV) and the immersive directional sounds. Time is also an important vector, since the action may shift place and direction during the piece.

Although innovative, this kind of content is still non-trivial to produce, in the sense that creators lack both the tools and the expertise to synthesise fully immersive, responsive and interactive $360^{\circ}$ videos that can provide these context-rich media pieces. These videos can be augmented with interactive information that allows the user to discover by itself the scenario (e.g., click in annotation or images attached to certain items in the $360^{\circ}$ scenario) or can have automatic pointers that lead the user to look in a certain way.

Our proposal addresses not only the content production pipeline (in particular the editing/annotation of $360^{\circ}$ video) but also the possibilities in terms of consumption of immersive videos, by proposing the exploration of new interaction metaphors with such multi-layered annotations and media sets (images, texts, inner videos). For this reason this paper explores two main issues in the interaction area: how the consumers navigate the video and, how the editor can overcome problems in the creation of the video.

On the consumer front, we propose several types of interactive annotations for $360^{\circ}$ videos, that will help the viewer locate and identify the points of interest, as well as define or select the type of information layers that they would like to focus on.

For the editors, the goal is to provide accessible tools for news professionals to have more control and at the same time freedom to edit and add relevant information to $360^{\circ}$ videos, by enabling features such as the definition and annotation of points of interest (both static and dynamic), captioning and adding multiple layers of information, including marketing and advertisement content, if adequate.

We expect that these innovations in both ends of the chain (creation and consumption) will foster better, more effective, more engaging and more economically attractive applications of this medium that is starting to find its way into the crowded space of journalism. 
For the visualisation of $360^{\circ}$ videos this research is essential to define new guidelines for the presentation of graphic and interactive content while allowing in-video edition of immersive content.

\section{A. Research Approach}

Based on the previously defined concepts and ideas, the main research topics that will be addressed in this paper are:

1) How to present stories and relevant information in $360^{\circ}$ videos?

2) How to develop an editing pipeline solution for journalists or users with video editing skills?

In order to address these questions, several annotation guidelines are depicted and a prototype video player was developed to present the editor's annotation pipeline.

\section{B. Document Structure}

In the following section we will present related work and important research that was considered in this project. In the third section, new ideas and guidelines for dynamic annotation will be addressed. The fourth section presents the workflow for the annotation along with a video player capable of rendering the annotated $360^{\circ}$ videos. The fifth section presents a fasttrack test to evaluate the proposed solution. The results are finally discussed in section VI. The conclusions discuss the current stage of the project and future directions.

\section{RELATED WORK}

While the consumer-oriented $360^{\circ}$ video content market has been growing more than ever [1], the concepts of immersive journalism and interactive video content are not exclusive to this decade. Significant work in these areas provides an interesting view of the future of journalists and news outlets [2]. Also, the multimedia convergence present in the communication science fields is conditioning both the craft and the content.

Current Virtual Reality (VR) headsets have become quite affordable in recent years and $360^{\circ}$ video as become much more disseminated. Much of the visualisation techniques used in $360^{\circ}$ video borrow ideas from the Augmented Reality (AR) world and can thus be used as an example.

\section{A. Virtual Reality}

Virtual Reality is a combination of different technologies, in which virtual environment rendering, interface, and system integration are key, and creating a virtual environment is a core element in virtual reality research [3]. Naturally, with a decline in consumer electronics production costs come very accessible devices. The Oculus Rift and Google cardboard are a few among the spectrum of consumer-centric head-mounted displays (HMD) [4].

On the software side, entertainment and scientific industries alike show interest in this technology. From production process simulations, to recreational or serious video games, the result is not simply a myriad of educational, training, simulation and entertainment tools, but also a very fleshed out vocabulary and knowledge base [5].

\section{B. Visualisation}

One can argue that despite the rapid advance in technology, there are still very few immersive VR applications outside research labs and novelty markets. Lack of usable and effective interaction and visualisation techniques are often the cause [6]. Much like graphics frameworks for 2D user interfaces emerged out of convenience and necessity [7], VR interaction metaphors will be isolated from application-specific tasks.

This modular approach aims to reduce the space of the problem, especially when one considers the similarity between VR and Augmented Reality (AR) setups in user-centric content. Outside of entertainment, VR content will tend towards realism as it is used in simulation, training and documentary tools. This real-world component is what allows VR interaction metaphors and visualization techniques to borrow from AR applications.

Many of the simplest AR systems [8] use the GPS position and the compass to track the location where the information will be rendered. The virtual information is usually displayed using the "bubble metaphor" [9], where the size of the bubble depends on relevance and the distance of the information to the user. This means that several labels located at a certain georeferenced place will be fighting for attention in the screen thus creating a confusing interface for the user.

Grasset et al. [10] propose a solution, which simplifies the visual clutter of the labels by positioning them in more visible places according to the content of the image. Examples of this type of interaction are the AR platforms Layar (http://www.layar.com) and Junaio (http://www.junaio.com). Other projects such as Liu et al. [11] explore the possibilities of automatic annotation of information based on algorithms of attention analysis of the video.

When one approaches a VR interface this way, one will find many of the same problems - and often the same solutions. One such example is the usage of viewport motion control techniques such as gaze-directed steering [12].

\section{Immersive Journalism}

Journalism as a social construct is evolving - the shift to new media formats is allowing the rationalisation of journalistic labour, but also creating a market for publishers to capitalise [13]. While experimental panoramic pictures and films are recorded throughout the history of imaging, only recently has technology caught up with the information volume demands.

Furthermore, the novelty of these types of technology generates a broad speculative ground in which nonspecialist content creators struggle to convey a narrative in a coherent and immersive manner [14].

While earlier studies on online journalism and ethics predicted that new, interactive platforms would bring out a new form of hybrid news content, the preferred area of cyber journalism today continues to be the news website. Despite limited to hypertext and simple links at first, the complexity of the Web allows these websites to deliver immersive multimedia content now more than ever [15]. 


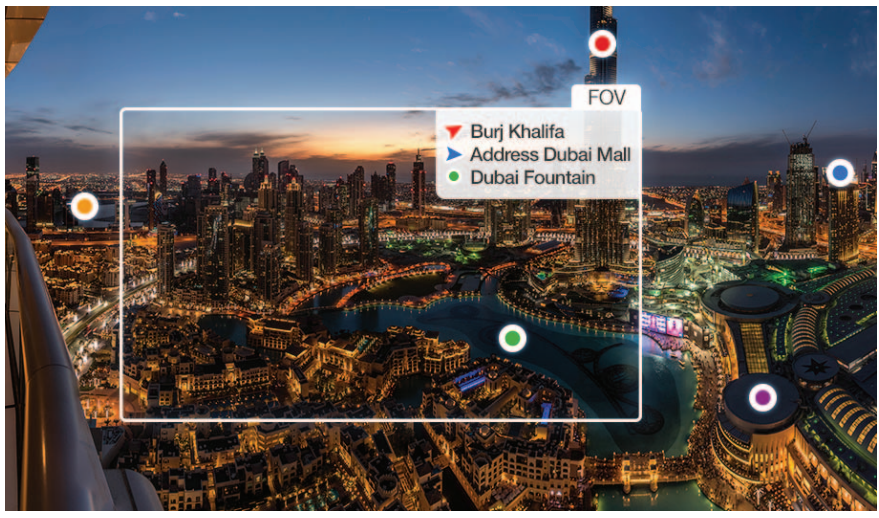

Fig. 1. POI listing aggregation concept. The idea is to present all the relevant locations listed together. The green dot is in the FOV while the others have arrows pointing to their location.

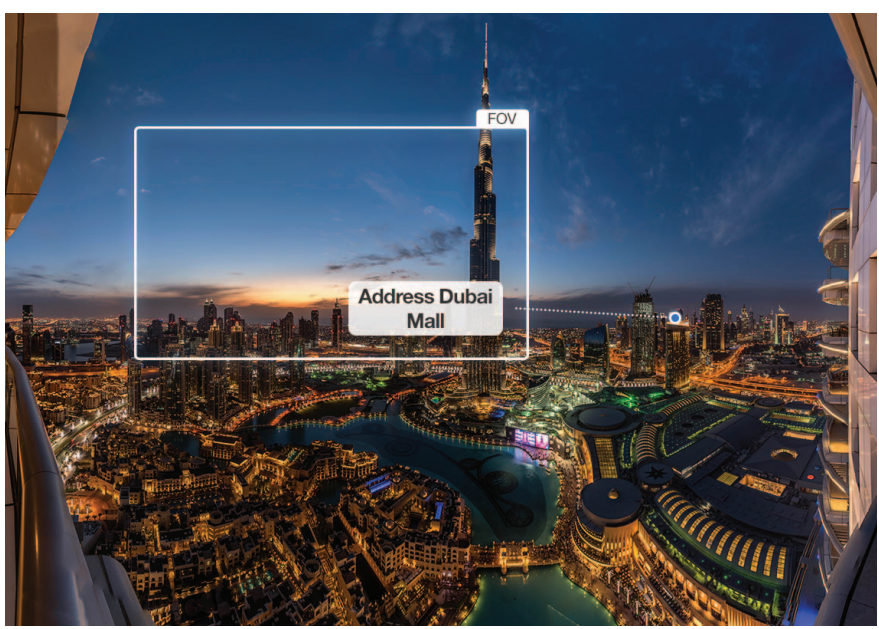

Fig. 2. Billboard and arrow usage concept. The Billboard is a board of information always facing the user. The arrow points to the location even if it is behind the user.

\section{DYNAMIC ANNOTATION}

After an analysis of pieces of content in the $360^{\circ}$ journalistic medium, potential visual ambiguity sources were detected for the final consumer. These regard the identification of visual points and locations, as well as readable subtitling in $360^{\circ}$ videos.

A list of potential visual annotation paradigms (not to be traditionally edited on top of currently existing video information but to be applied at run-time) was considered, from which two paradigms are already developed as a prototype with clips from existing $360^{\circ}$ video news content (see next section). We believe that with the addition of dynamic information that follows the user's visual field, it is possible to mitigate ambiguities regarding location information in the space of a $360^{\circ}$ video, as well as improving general comprehension of the video content by the user. The list of visualisation paradigms that were considered include:

1) Media information display [16], techniques to present data:
Billboards the most common paradigm [9], a squared area will be superimposed to the scene above a certain area or displaced with an arrow pointing to the POI. Inside the square all sorts of multimedia content can be inserted from text to images, videos or mini-webpages;

POI listing aggregation summary of all the points-ofinterest (POI) in the FOV or close to the user, ordering them by proximity (Figure 1);

Static subtitling this is the traditional subtitling mode where the text (or media content) is shown in the same place in the screen (or FOV) independently of where the user is looking;

Oracle and Ticker following the paradigm in news channels, the ticker here would be applied to the $360^{\circ}$ space;

2) Narrative and attention focusing, techniques to tell a story:

Arrows a wide range of arrows can be added to the information to highlight events happening outside the FOV. The goal is to induce the eye gaze of the user to focus on a certain scene item or event (Figure 2);

Map and Compass the visualisation of a map with a compass can help in the exploration of the 360-degree scene. Animated highlights can indicate events happening outside the FOV or trace a suggested path for the user to look;

It should be noted that contrary to Augmented Reality (AR) applications - that feature real-time object identification from videos - the proposed solution will rely on pre-processed, completely authored annotations of places and objects. This not only gives the content creator more control over the user's experience but also provides a deterministic framework for the consumer perspectives. Nevertheless the same solution could be extended to real-time scenarios, if tracking was available.

\section{Interactive $360^{\circ}$ VIDEO Player PROTOTyPe}

In this section we present the workflow of the editor, from the annotation to the visualisation of the $360^{\circ}$ video. Before experimenting with the aforementioned visualisation paradigms we created a fully customisable video player for $360^{\circ}$ videos.

The prototype $360^{\circ}$ video player capable of displaying augmented stereoscopic imagery in a VR environment was designed and implemented using the Unity 3D engine (http://www.unity3d.com). The player works by opening both a $360^{\circ}$ video file and a modified .srt subtitle file, and rendering the content and annotations simultaneously in a VR environment. While special annotations are placed on various points of the screen, regular subtitles from the .srt file are also featured. These are presented at the bottom of the user's viewport and follow their gaze.

This application was designed to run on an Oculus Rift Development Kit 2 (DK2) HMD device.

While content playback with reasonable fidelity was a concern, it became necessary to also develop authoring tools 


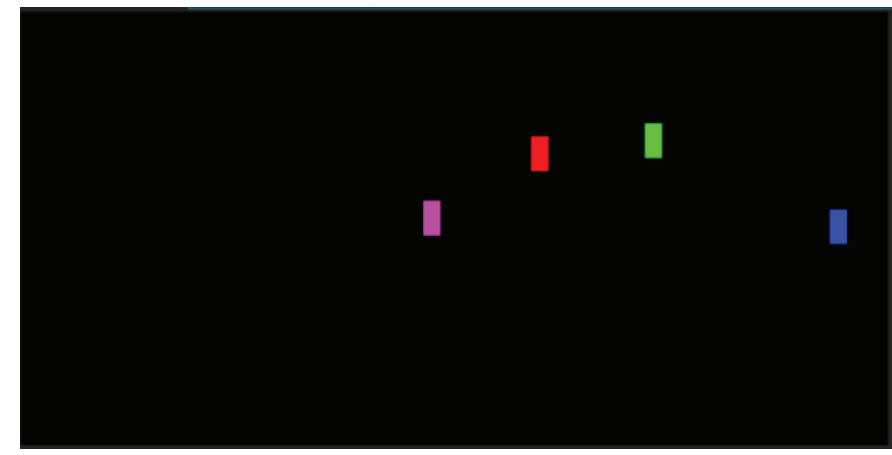

Fig. 3. Annotation video mask. This is a single frame of the video depicting four annotated locations. This mask can be created using a layer in any common video editor software.

that allow for fast and intuitive iteration. The conceived system integrates a pipeline capable of generating human-readable annotation files. This is a one-time process and is done in two steps:

1) A video mask is rendered by the user, composed of coloured spots on a black background, each of which corresponds to an annotation and its position and timing on the video (Figure 3). This type of video can be easily created using video editing software. Using the screen blending mode (featured in most mainstream editors) allows a transparent layer overlap of content and mask, thus allowing to paint actual objects on top of the video.

2) This video mask and an optional regular subtitle file are parsed in a computer vision algorithm (Figure 4) that generates an augmented srt subtitle file compatible with the prototype. This does not modify the original files in any way.

This type of offline parsing reduces the necessary file size for augmented playback, while also allowing regular playback and obeying .srt format standards.

To explore the paradigms devised in the previous section we selected two main types of annotation for our prototype. The implemented paradigms - Billboards and Arrows - were selected on the basis of their applicability to journalistic pieces. Namely, billboards and arrows are applied to multiplePOI experiences (Figure 5), but can also effectively direct the user's attention to a single moving POI (Figure 6).

\section{TESTING AND Evaluation}

In this test we detail the experiments used to evaluate the prototype. The goal was to provide insights by using a fast track test. Validation of the proposed method was based on the results of a questionnaire used to retrieve data from the user tests.

By dividing the presentation into two parts the tests demand less from the users' attention span as they can focus on answering the questions that concern the section they have last seen. Additionally, the questionnaire was made short and explicit, focusing on the users' personal experience.

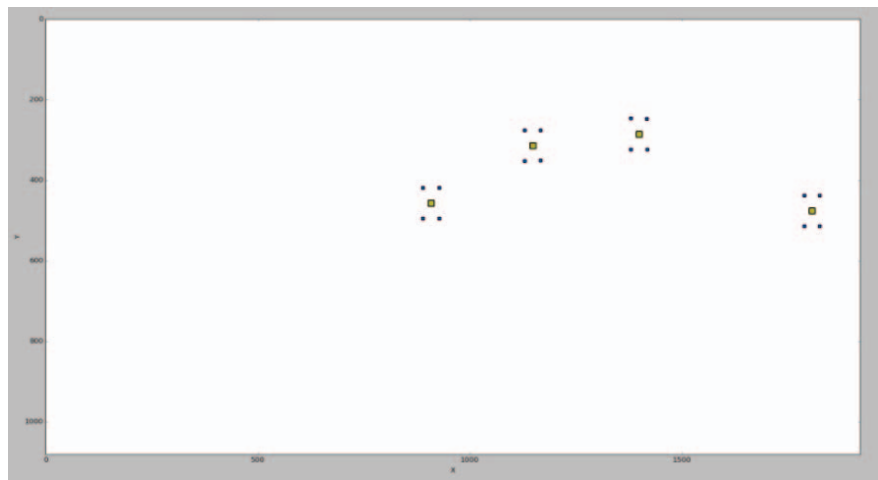

Fig. 4. Video mask tracking inside computer vision algorithm. Automatic process for point detection.

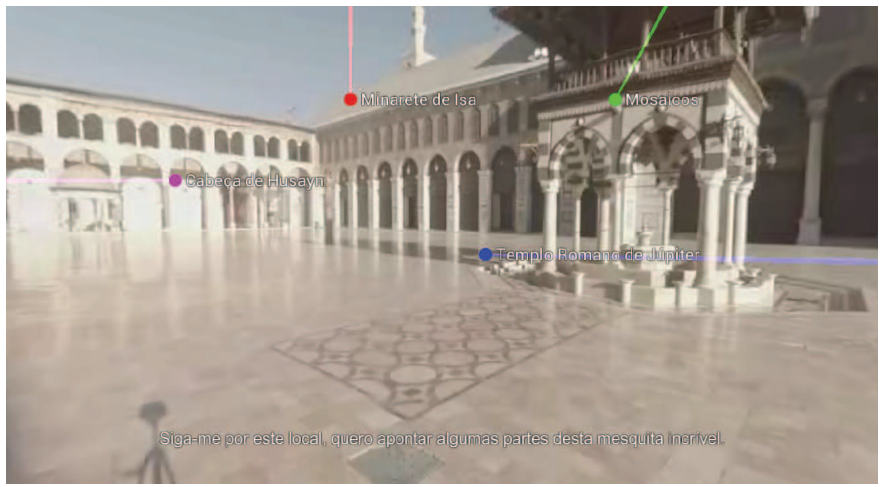

Fig. 5. Augmented $360^{\circ}$ video playing with annotations featuring multiple billboards. The blue and violet points are behind the user, but displayed to the right or left to guide facing.

Ultimately, the questionnaire was meant to ascertain the level of understanding the user had regarding the news. This level of understanding refers to the ability of identifying points of interest and gaze in a scene. Some verbal feedback occurred, spontaneously, from the users, after the experience.

\section{A. Experiment Part I - Inside Syria}

The excerpt of the "Inside Syria" documentary (ABC News) had four locations annotated, using the billboard technique, as presented in Figure 5. All of these locations, as well as the position of the viewer are fixed. The participant will view both the original (annotation-free) and the annotated version of the excerpt.

\section{B. Experiment Part II - The Contenders}

The excerpt of the "The Contenders" documentary had one feature of interest (Bernie Sanders, an American politician (virtualitylab.com)) annotated, using a single billboard, as shown in Figure 6. Although the position of the viewer is fixed, the position of the annotation follows the motion of the feature of interest. The participant will view both the annotated and unadulterated version of the excerpt. 


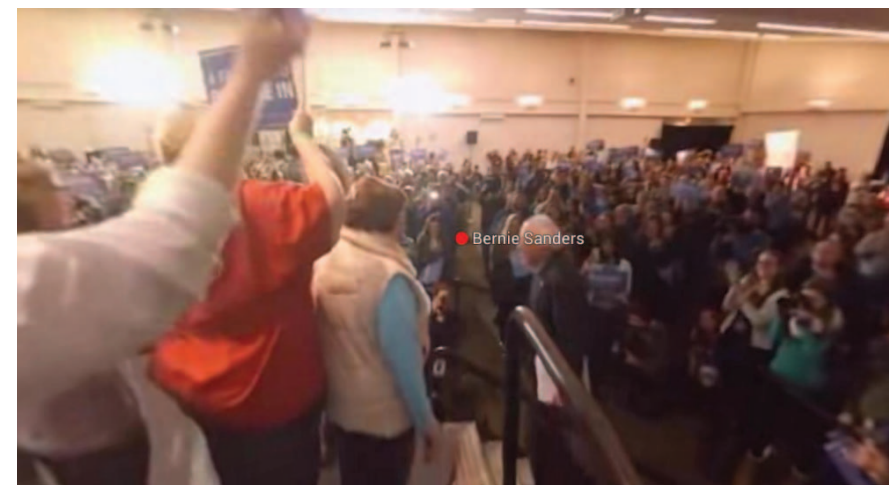

Fig. 6. Augmented $360^{\circ}$ video playing with annotations featuring a single billboard. The annotation is directly in the user's view.

\section{User Test Protocol}

The tests did not exceed ten minutes in individual duration, on average. The protocol of those tests was as follows:

1) Open new questionnaire and do pre-experience questions (Figure 7).

2) Present to the user the experimental context, explaining the idea behind dynamic annotations and indicating that the video will be displayed twice (the second time with dynamic annotations).

3) Place the Oculus Rift DK2 HMD in a position that avoids light from leaking into it, is comfortable and allows for a maximum feeling of presence. This step requires the user to give feedback.

4) Place the headphones.

5) Start playing the first part "Inside Syria" (both with no annotations and dynamic annotations).

6) Inform the user the questionnaire must now be answered to, up until an empty section designated as "Pause".

7) Start playing the second part "The Contenders" (both with no annotations and dynamic annotations).

8) Inform the user that the rest of the questionnaire must now be answered.

9) Accept possible verbal feedback from the user.

All users followed the above protocol. Some users reported difficulty in reading and seeing graphical elements without their prescription glasses, which required replaying the part in question. Other users reported discomfort during the experiment which required readjustments to be made before restarting the experiment.

The questionnaire followed a Likert scale (with five options, ranging from "Totally Disagree" to "Fully Agree") and "Yes/No" formats for its possible answers. The questionnaire was built using Google Forms for its ease of use, web storage and exporting possibilities.

\section{Questionnaire}

These were the questions presented to the participants, relative only to the first part of the experiment.

Q1 Without annotations, I felt difficulty in identifying the locations referred by the journalist.

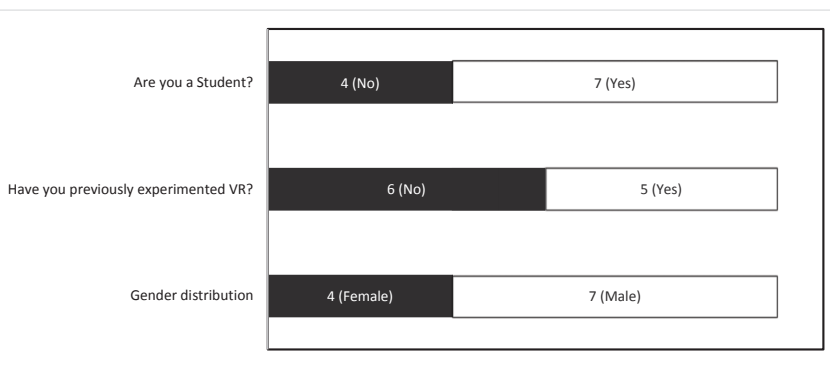

Fig. 7. Pre-experience questions.

Q2 With annotations, I felt difficulty in identifying the locations referred by the journalist.

Q3 Annotations were a distraction.

Q4 I understood the topic of the excerpt.

Q5 The subtitles were easy to read.

Q6 The subtitles were a distraction.

Q7 With annotations, how many locations were you able to identify? (\#Number)

The following questions concern the second part of the experiment.

Q8 Without annotations, I felt difficulty in focusing on the action.

Q9 With annotations, I felt difficulty in focusing on the action.

Q10 Annotations were a distraction.

Q11 I understood the topic of the excerpt.

Q12 The subtitles were easy to read.

Q13 The subtitles were a distraction.

\section{RESUlTS AND DISCUSSION}

This section contains a descriptive statistical analysis of the group of participants as well as the results of both parts of the experiment.

A total number of 11 individuals participated in the experiment. As seen in Figure 8, most users had between 20 and 30 years. The youngest individual was 13 years old, whereas the oldest was 63. Most individuals were students and some had already experienced virtual reality. There were four females and seven males in the group (see Figure 7).

\section{A. Experiment Part I - Inside Syria}

Table I condenses the number of answers, using the Likert scale, given to the aforementioned questions.

Comparing the answers to Q1 with the answers given to Q2, it is possible to see that participants did find it easier to identify the locations with annotations versus with no annotations. However, regarding Q3, several participants claimed the annotations are a distraction. Most participants had no issue in understanding the topic of the excerpt (9 in 11), as seen by their answers to $\mathrm{Q} 4$. There is no consensus regarding the readability of the subtitles (Q5). Verbal feedback, given by some of the participants, suggested that the excessively low position of the subtitles in the field of view was detrimental to 


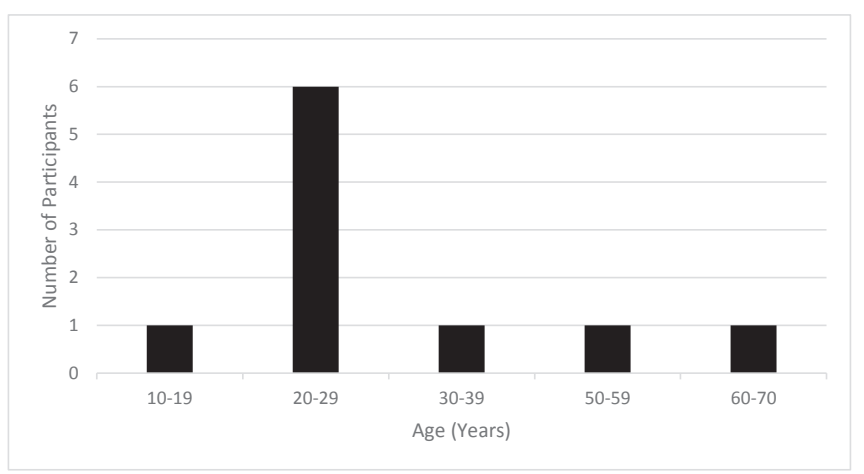

Fig. 8. Participants' age distribution.

TABLE I

QUESTIONNAIRE RESULTS (PART I - INSIDE SYRIA)

\begin{tabular}{cccccc}
\hline \multirow{2}{*}{ Question } & \multicolumn{5}{c}{ Answer } \\
\cline { 2 - 6 } & 1 & 2 & 3 & 4 & 5 \\
\hline Q1 & 1 & 4 & 1 & 4 & 1 \\
Q2 & 4 & 5 & 1 & 1 & 0 \\
Q3 & 2 & 1 & 3 & 5 & 0 \\
Q4 & 0 & 2 & 0 & 2 & 7 \\
Q5 & 3 & 3 & 0 & 3 & 2 \\
Q6 & 4 & 3 & 3 & 1 & 0 \\
\hline
\end{tabular}

its readability. Lack of contrast between text and content was also mentioned. Most participants did not find the subtitles to be distracting, with only one mildly finding them to be intrusive. Some users reported ignoring the subtitles altogether, and focused only on the audio content.

TABLE II

ANSWERS TO QUESTION Q7

\begin{tabular}{cc}
\hline Number of identified locations & Number of Answers \\
\hline 3 & 2 \\
4 & 7 \\
5 & 1 \\
6 & 1
\end{tabular}

Finally, Q7's goal was to ascertain if the participants were able to retain the number of annotated locations. Although most (seven), were able to correctly remember the number of marked locations, the rest of the users either overestimated or underestimated said number. This discrepancy could indicate that some users may have been confused, or, more likely, were not paying attention to the number of locations they were seeing in the video, as they reported in Q2, that they generally had no problem in identifying the annotated locations. Some participants reported difficulty in remembering the number of locations and confusion due to the number and colour of the lines in their field of view (consistent with some of the answers provided to Q3).

\section{B. Experiment Part II - The Contenders}

TABLE III

Questionnaire Results (PART II - THE CONTENDERS)

\begin{tabular}{rlllll}
\hline \multirow{2}{*}{ Question } & \multicolumn{5}{c}{ Answer } \\
\cline { 2 - 6 } & 1 & 2 & 3 & 4 & 5 \\
\hline Q8 & 1 & 0 & 3 & 4 & 3 \\
Q9 & 8 & 2 & 0 & 1 & 0 \\
Q10 & 7 & 2 & 2 & 0 & 0 \\
Q11 & 0 & 1 & 1 & 2 & 7 \\
Q12 & 2 & 3 & 0 & 4 & 2 \\
Q13 & 6 & 3 & 2 & 0 & 0 \\
\hline
\end{tabular}

Table III condenses the number of answers, using the Likert scale, given to the aforementioned questions.

Most users agreed with the statement presented in Q8. Only one user completely disagreed with it. Some reported having looked around in several directions, during the excerpt with no annotations, as they were unable to identify the candidate in their field of view. Nearly all of the participants found no difficulty in following the action in the excerpt with annotations, with only one reporting a mild level of difficulty (Q9). No user found the annotations to be distracting, with most (seven) users completely disagreeing with the statement of Q10. Again, most users had no difficulty in understating the subject of the excerpt, with only one user reporting some difficulty, in Q11. This could be due to the fact that this second excerpt had less spoken information. Similar to what happened in Q5, there is no consensus regarding the readability of the subtitles as seen by the answers to Q12. Contrary to what happened in the first part of the experiment, no users found the subtitles to be distracting.

\section{CONCLUSIONS}

The contributions of this work, in helping developing annotation techniques for $360^{\circ}$ videos, have been presented. In this paper a set of visual paradigms for video annotation and narrative storytelling have been proposed and two of them were implemented and tested. The contribution of these techniques to the immersive journalism field were also defined and highlighted.

The implemented $360^{\circ}$ video player prototype demonstrates a possible means of implementing two of the proposed annotation paradigms, the billboards and arrows, and using them for augmenting $360^{\circ}$ videos. The two-part experiment provided insight into determining the contributions of this work, from the content consumers' perspective. Participants reported being capable of identifying locations and accompanying the action of these videos with greater ease, when comparing to their experience with no annotations. However, some considered the annotations to be somewhat distracting, particularly in a situation where multiple billboards were present. As such, the advantages of the proposed annotation techniques should be 
considered as a possible solution to some of the problems viewers have in $360^{\circ}$ videos.

\section{A. Future Work}

The present work opens several lines of research. The feedback given during the experiment shows that this methodology of dynamic annotations is potentially beneficial to the viewers' experience and, as such, should be further explored. Specifically, the implementation and testing of additional dynamic annotation techniques is to be made. Regarding the annotation techniques already developed, the billboard and arrows, the results show that they can still be improved, as some viewers considered them obtrusive or confusing, particularly when multiple annotations were present. Regarding the subtitles, the tests were inconclusive, although improving their readability and position could eventually improve the opinions of the viewers to an overall positive result. As this is a very recent development for both UI (User Interface) and UX (User Experience), the area of immersive journalism could benefit from the creation of a universal "styles guide" that would allow for the conscious creation of content that is to be annotated and augmented. The popularity of emerging technologies and possibilities could lead to the reinvention of journalism and its practises.

\section{ACKNOWLEDGMENTS}

This work has been financed by FourEyes, "TEC4Growth Pervasive Intelligence, Enhancers and Proofs of Concept with Industrial Impact/NORTE-01-0145-FEDER-00002”, financed by the North Portugal Regional Operational Programme (NORTE 2020), under the PORTUGAL 2020 Partnership Agreement, and through the European Regional Development Fund (ERDF). The authors would also like to acknowledge the support in terms of infrastructures and technologies from the "MASSIVE Multimodal Acknowledgeable multiSenSorial Immersive Virtual Enviroments" project (REC I/EEISII/0360/2012 - COMPETE, QREN and FEDER).

\section{REFERENCES}

[1] Martin Kesselman. 2016 Consumer Electronics Show, Las Vegas: Virtual 3D Cameras and More. Library Hi Tech News, 33(3):16-19, may 2016.
[2] Anabela Gradim. O jornalista multimédia do século XXI. Informação e Comunicação Online, 1:117-134, 2003.

[3] Ning-Ning Zhou and Yu-Long Deng. Virtual reality: A state-of-the-art survey. International Journal of Automation and Computing, 6(4):319325, nov 2009.

[4] Wendy Anne Powell, Vaughan Powell, Phillip Brown, Marc Cook, and Jahangir Uddin. Getting around in Google Cardboard - exploring navigation preferences with low cost mobile VR. 2016.

[5] M. Zyda. From visual simulation to virtual reality to games. Computer, 38(9):25-32, sep 2005.

[6] Douglas A. Bowman. Interaction techniques for common tasks in immersive virtual environments: design, evaluation, and application. 1999.

[7] James D. Foley. A standard computer graphics subroutine package. Computers \& Structures, 10(1):141-147, 1979.

[8] Feng Zhou, H. B. L. Duh, and M. Billinghurst. Trends in augmented reality tracking, interaction and display: A review of ten years of ismar. In Mixed and Augmented Reality, 2008. ISMAR 2008. 7th IEEE/ACM International Symposium on, pages 193-202, Sept 2008.

[9] Yuichiro Takeuchi and Ken Perlin. Clayvision: The (elastic) image of the city. In Proceedings of the SIGCHI Conference on Human Factors in Computing Systems, CHI '12, pages 2411-2420, New York, NY, USA, 2012. ACM

[10] Raphael Grasset, Tobias Langlotz, Denis Kalkofen, Markus Tatzgern, and Dieter Schmalstieg. Image-driven view management for augmented reality browsers. In Proceedings of the 2012 IEEE International Symposium on Mixed and Augmented Reality (ISMAR), ISMAR '12, pages 177-186, Washington, DC, USA, 2012. IEEE Computer Society.

[11] Huiying Liu, Shuqiang Jiang, Qingming Huang, and Changsheng Xu. A generic virtual content insertion system based on visual attention analysis. In Proceedings of the 16th ACM International Conference on Multimedia, MM '08, pages 379-388, New York, NY, USA, 2008. ACM.

[12] Cristiano Carvalheiro, Rui Nóbrega, Hugo da Silva, and Rui Rodrigues. User Redirection and Direct Haptics in Virtual Environments. In Proceedings of the 2016 ACM on Multimedia Conference - MM '16, pages 1146-1155, New York, New York, USA, 2016. ACM Press.

[13] Leopoldina Fortunati, Mauro Sarrica, John O'Sullivan, Aukse Balcytiene, Halliki Harro-Loit, Phil Macgregor, Nayia Roussou, Ramón Salaverría, and Federico de Luca. The Influence of the Internet on European Journalism. Journal of Computer-Mediated Communication, 14(4):928-963, jul 2009.

[14] John Vernon Pavlik. Journalism and New Media. page 246, 2001.

[15] Ricardo Cruz and Ricardo Fernandes. Journalism in Virtual Worlds. Journal For Virtual Worlds Research, 4(1), 2011.

[16] J. V. Pavlik and F. Bridges. The Emergence of Augmented Reality (AR) as a Storytelling Medium in Journalism. Journalism \& Communication Monographs, 15(1):4-59, jan 2013. 